\title{
WINDING BEHAVIOUR OF FINITE-TIME SINGULARITIES OF THE HARMONIC MAP HEAT FLOW *
}

\author{
Peter Topping
}

\begin{abstract}
We settle a number of questions about the possible behaviour of the harmonic map heat flow at finite-time singularities. In particular, we show that a type of nonuniqueness of bubbles can occur at finite time, we show that the weak limit of the flow at the singular time can be discontinuous, we determine exactly the (polynomial) rate of blow-up in one particular example, and we show that 'winding' behaviour of the flow can lead to an unexpected failure of convergence when the flow is (locally) lifted to the universal cover of the target manifold.
\end{abstract}

\section{Introduction}

Given a compact Riemannian manifold $\mathcal{N}$, we define the energy of a sufficiently smooth map $u: D \rightarrow \mathcal{N}$ from the 2 -disc $D$ to be

$$
E(u)=\frac{1}{2} \int_{D}|d u|^{2}
$$

(The integral over an alternative region $\Omega \subset \mathbb{R}^{2}$ will be denoted by $E(u, \Omega)$.) The tension field $\tau=\tau(u) \in \Gamma\left(u^{*}(T \mathcal{N})\right)$ is the vector field along $u$ representing minus the $L^{2}$-gradient of $E$. If we write this as $\tau^{i} \frac{\partial}{\partial y^{i}}(u)$ in terms of local coordinates $\left\{y^{i}\right\}$ on $\mathcal{N}$ then we have the expression

$$
\tau^{i}=\Delta u^{i}+\Gamma_{k l}^{i}(u) \nabla u^{k} \cdot \nabla u^{l}
$$

where $\Gamma_{k l}^{i}$ are the Christoffel symbols for the target.

Although this intrinsic viewpoint will be optimal when we are dealing with smooth maps into $\mathcal{N}$ (including most of Sections 3 and 4) for much of this paper we will consider maps into $\mathcal{N}$ to have been composed with a fixed isometric embedding $\mathcal{N} \hookrightarrow \mathbb{R}^{N}$. (This simplifies some calculations, and

*This draft: Feb. 2003. Partly supported by an EPSRC Advanced Research Fellowship 
the discussion of Sobolev spaces and weak solutions.) We then see the tension as a vector field in the ambient $\mathbb{R}^{N}$, giving it the simple expression

$$
\tau=(\Delta u)^{T}
$$

where the superscript $T$ means the projection onto $T_{u(x)} \mathcal{N}$.

In this paper we will consider the harmonic map flow from $D$ to $\mathcal{N}$. This is a time-dependent solution $u: D \times[0, T] \rightarrow \mathcal{N}$ of the harmonic map heat equation of Eells-Sampson [6]

$$
\frac{\partial u}{\partial t}=\tau(u(t))
$$

where $u(t):=u(\cdot, t)$ is the restriction (or more generally the trace) of $u$ to the time slice $D \times\{t\}$. At times $t$ at which the flow is smooth, the energy of the flow decays according to

$$
\frac{d}{d t} E(u(t))=-\int_{D}|\tau(u(t))|^{2} .
$$

Since we are working with a two dimensional domain, the energy is conformally invariant (in particular the energy of a map is preserved under precomposition with a dilation) and we have a theory of Struwe at our disposal which includes the following existence theorem. (See also Chang [2] where the situation in which the domain has boundary is explicitly considered.)

Theorem 1.1 (Struwe [15].) Given $u_{0} \in C^{\infty}(\bar{D}, \mathcal{N})$, there exists a weak solution $u \in W_{\text {loc }}^{1,2}(D \times$ $[0, \infty), \mathcal{N})$ to $(1.2)$ which is smooth in $\bar{D} \times[0, \infty)$ except possibly at finitely many singular points in $\bar{D} \times(0, \infty)$, and has the following properties:

(a) $u(0) \equiv u_{0}$;

(b) $\left.\left.u(\cdot, t)\right|_{\partial D} \equiv u_{0}\right|_{\partial D}$ for all $t \geq 0$;

(c) $E(u(t))$ is a (weakly) decreasing function of $t$ on $[0, \infty)$;

(d) If the flow is smooth for $t \in[0, T)$, then it is the unique smooth solution over this time interval with the given boundary and initial data;

(e) If $(x, T) \in \bar{D} \times(0, \infty)$ is a singular point, then energy concentrates in the sense that

$$
\lim _{\nu \downarrow 0} \limsup _{t \uparrow T} E\left(u(t), D_{\nu}(x) \cap D\right) \neq 0 .
$$

We call this flow the 'Struwe solution' or 'Struwe flow.'

Here we have denoted by $D_{\nu}(x)$ the open disc in $\mathbb{R}^{2}$ centred at $x \in \mathbb{R}^{2}$ and of radius $\nu>0$. We will also be using the abbreviation $D_{\nu}:=D_{\nu}(0)$ and of course $D=D_{1}$. 
Whilst the Struwe solution is unique amongst weak solutions satisfying conditions (a) to (c) of the theorem - see Freire [7] - there are now known, in certain cases, to be other weak solutions satisfying (a) and (b) but not (c) with similar regularity properties to the Struwe solution - see [19] and [1].

Given a flow $u$ from Theorem 1.1, we may integrate (1.3) and use the fact that $E(u(t))$ is weakly decreasing, to get the useful well-known bound on the tension

$$
\int_{0}^{\infty}\|\tau(u(t))\|_{L^{2}(D)}^{2} d t \leq E\left(u_{0}\right)<\infty
$$

Whilst Struwe's Theorem 1.1 allows the possibility of some finite-time singularities, it was not clear at the time whether or not such singularities could occur. Later, Chang-Ding-Ye [3] constructed an example of a flow in which a singularity is forced to occur at finite time. Their construction relies on a certain corotational symmetry which is required to invoke maximum principle techniques. However, work of Qing-Tian [12] (included in Theorem 1.4 below) opens up the possibility of more robust methods as we now describe. In 1978, Lemaire proved the following useful theorem.

Theorem 1.2 (Lemaire [8].) Any harmonic map from the 2-disc D to $\mathcal{N}$ which is constant on the boundary, must be constant throughout D.

A corollary of this result is that no nontrivial homotopy class of maps from $D$ which has fixed, constant boundary values, can contain a harmonic map. In an appendix to this paper we sketch a proof of the existence of a homotopy class of maps which not only fails to contain a harmonic map, but does so in a quantified way below a certain energy level:

Lemma 1.3 There exist a compact target manifold $\mathcal{N}$, a smooth map $v_{0}: D \rightarrow \mathcal{N}$ and $\varepsilon>0$ such that every smooth map $v: D \rightarrow \mathcal{N}$ homotopic to $v_{0}$ fails to be harmonic, and if $E(v) \leq E\left(v_{0}\right)$ also, then

$$
\int_{D}|\tau(v)|^{2} \geq \varepsilon
$$

This lemma, coupled with (1.3) and the fact that $E \geq 0$ immediately implies that no heat flow starting with an initial map $u_{0}$ homotopic to $v_{0}$ and with $E\left(u_{0}\right) \leq E\left(v_{0}\right)$ can be smooth beyond time $t=\frac{E\left(u_{0}\right)}{\varepsilon} \leq \frac{E\left(v_{0}\right)}{\varepsilon}$. This obviously gives a rich source of robust examples of finite-time singularities. Note that we cannot perturb away a finite-time singularity here by adjusting the initial map, although dependency of the singular points on the initial map is not addressed. The general principle behind the proof of this lemma will be exploited later on, in a concrete situation, to force certain flows with special properties to blow up in finite time.

The original work of Struwe also gave a basic description of what happens at a singularity; 'bubbling' in a similar form to that discovered by Sacks-Uhlenbeck [13] occurs. Gradually a better picture has been built up, by better understanding the properties of maps with small tension in $L^{2}$. The 
main tool, combining work of Struwe [15], Ding-Tian [4] (see also Qing [10] and Wang [21]) and Qing-Tian [12] (see also Lin-Wang [9]) is:

Theorem 1.4 Suppose that $u_{n}: D \rightarrow \mathcal{N} \hookrightarrow \mathbb{R}^{N}(n \in \mathbb{N})$ is a sequence of smooth maps which satisfy $E\left(u_{n}\right)<M$ for some constant $M$, and all $n \in \mathbb{N}$, and $\tau\left(u_{n}\right) \rightarrow 0$ in $L^{2}(D)$ as $n \rightarrow \infty$.

Then we may pass to a subsequence in $n$, and find a harmonic map $u_{\infty}: D \rightarrow \mathcal{N}$, and a set $\left\{x^{1}, \ldots, x^{m}\right\} \subset D$ such that

(a) $u_{n} \rightarrow u_{\infty}$ weakly in $W^{1,2}(D, \mathcal{N})$,

(b) $u_{n} \rightarrow u_{\infty}$ strongly in $W_{l o c}^{2,2}\left(D \backslash\left\{x^{1}, \ldots, x^{m}\right\}, \mathcal{N}\right)$.

Moreover, for each $l \in\{1, \ldots, m\}$, there exists $k=k(l) \in \mathbb{N}$ such that for $i \in\{1, \ldots, k\}$ there exist sequences $a_{n}^{i} \rightarrow x^{l} \in D$ and $\lambda_{n}^{i} \downarrow 0$ as $n \rightarrow \infty$, and nonconstant harmonic maps $\omega^{i}: S^{2} \rightarrow \mathcal{N}$ (which we precompose with inverse stereographic projection to view them as maps $\mathbb{R}^{2} \cup\{\infty\} \rightarrow \mathcal{N}$ ) such that:

(i)

$$
\frac{\lambda_{n}^{i}}{\lambda_{n}^{j}}+\frac{\lambda_{n}^{j}}{\lambda_{n}^{i}}+\frac{\left|a_{n}^{i}-a_{n}^{j}\right|^{2}}{\lambda_{n}^{i} \lambda_{n}^{j}} \rightarrow \infty,
$$

as $n \rightarrow \infty$, for each unequal $i, j \in\{1, \ldots, k\}$.

(ii)

$$
\lim _{\nu \downarrow 0} \lim _{n \rightarrow \infty} E\left(u_{n}, D_{\nu}\left(x^{l}\right)\right)=\sum_{i=1}^{k} E\left(\omega^{i}\right) .
$$

(iii)

$$
u_{n}(x)-\sum_{i=1}^{k}\left(\omega^{i}\left(\frac{x-a_{n}^{i}}{\lambda_{n}^{i}}\right)-\omega^{i}(\infty)\right) \rightarrow u_{\infty}(x),
$$

as functions of $x$ from $D_{\nu}\left(x^{l}\right)$ to $\mathcal{N} \hookrightarrow \mathbb{R}^{N}$ (for sufficiently small $\nu>0$ ) both in $W^{1,2}$ and $L^{\infty}$.

(iv) For each $i \in\{1, \ldots, k\}$ there exists a finite set of points $\mathcal{S} \subset \mathbb{R}^{2}$ (which may be empty, but could contain up to $k-1$ points) with the property that

$$
u_{n}\left(a_{n}^{i}+\lambda_{n}^{i} x\right) \rightarrow \omega^{i}(x),
$$

in $W_{l o c}^{2,2}\left(\mathbb{R}^{2} \backslash \mathcal{S}, \mathcal{N}\right)$ as $n \rightarrow \infty$.

We refer to the map $u_{\infty}: D \rightarrow \mathcal{N}$ as a 'body' map, and the (smooth) maps $\omega^{i}: S^{2} \rightarrow \mathcal{N}$ as 'bubbles' or 'bubble' maps. The points $\left\{x^{1}, \ldots, x^{m}\right\}$ will be called 'bubble points,' and the $\lambda_{n}^{i}$ 'bubble scales.' 
Remark 1.5 Encoded in the $L^{\infty}$ convergence of part (iii) of Theorem 1.4 is the fact that at each bubble point, the union of the images of the body map $u_{\infty}$ and the bubbles $\omega^{i}$ is a connected set. (See Qing-Tian [12].)

Using Theorem 1.4, and its earlier versions, the authors mentioned above (Struwe, Ding, Tian, Qing, Wang, Lin) have described partly the bubbling which occurs at finite time in the harmonic map flow. We need a slight improvement of those results incorporating better control on the relationship between the bubble scales, the tension and the time left until the singularity. The following will be proved in Section 2 based on Theorem 1.4 above.

Theorem 1.6 Suppose $u \in W_{\text {loc }}^{1,2}(D \times[0, \infty), \mathcal{N})$ is a Struwe flow from Theorem 1.1, and $T \in(0, \infty)$ is a singular time. Then there exist times $t_{n} \uparrow T$ with the property that

$$
\left\|\tau\left(u\left(t_{n}\right)\right)\right\|_{L^{2}(D)}^{2}\left(T-t_{n}\right) \rightarrow 0
$$

as $n \rightarrow \infty$. Moreover, for every singular point $(x, T) \in D \times(0, \infty)$ at time $T$, there exist $k \in \mathbb{N}$ and, for each $i \in\{1, \ldots, k\}$, sequences $a_{n}^{i} \rightarrow x \in D$ and $\lambda_{n}^{i} \downarrow 0$ as $n \rightarrow \infty$, and nonconstant harmonic maps $\omega^{i}: S^{2} \rightarrow \mathcal{N}$ (which we view as maps $\mathbb{R}^{2} \cup\{\infty\} \rightarrow \mathcal{N}$ ) such that:

(i)

$$
\frac{\lambda_{n}^{i}}{\lambda_{n}^{j}}+\frac{\lambda_{n}^{j}}{\lambda_{n}^{i}}+\frac{\left|a_{n}^{i}-a_{n}^{j}\right|^{2}}{\lambda_{n}^{i} \lambda_{n}^{j}} \rightarrow \infty,
$$

as $n \rightarrow \infty$, for each unequal $i, j \in\{1, \ldots, k\}$.

(ii)

$$
\lim _{\eta \downarrow 0} \lim _{t \uparrow T} E\left(u(t), D_{\eta}(x)\right)=\lim _{t \uparrow T} E\left(u(t), D_{(T-t)^{\frac{1}{2}}}(x)\right)=\sum_{i=1}^{k} E\left(\omega^{i}\right) .
$$

(iii)

$$
u\left(t_{n}\right)-\sum_{i=1}^{k}\left(\omega^{i}\left(\frac{\cdot-a_{n}^{i}}{\lambda_{n}^{i}}\right)-\omega^{i}(\infty)\right) \rightarrow u(T),
$$

in $W^{1,2}\left(D_{\nu}(x), \mathcal{N}\right)$ (for sufficiently small $\left.\nu>0\right)$.

(iv) For each $i \in\{1, \ldots, k\}$ there exists a finite set of points $\mathcal{S} \subset \mathbb{R}^{2}$ (which may be empty, but could contain up to $k-1$ points) with the property that

$$
u\left(a_{n}^{i}+\lambda_{n}^{i} y, t_{n}\right) \rightarrow \omega^{i}(y),
$$

as functions of $y$, in $W_{\text {loc }}^{2,2}\left(\mathbb{R}^{2} \backslash \mathcal{S}, \mathcal{N}\right)$ as $n \rightarrow \infty$.

(v) For each $i \in\{1, \ldots, k\}$, we have

$$
\lambda_{n}^{i}\left(T-t_{n}\right)^{-\frac{1}{2}} \rightarrow 0
$$


Remark 1.7 Part (ii), in addition to being a so-called "no loss of energy" statement, now includes important information about the rate of blow-up of any singularity in the uniform limit $t \uparrow T$. Restricting to the bubbling at times $\left\{t_{n}\right\}$ then gives part $(\mathrm{v})$. (This rate of blow-up is well-known and may be established by various methods.) It is possible to improve this rate slightly to, say,

$$
\lambda_{n}^{i}\left[\left(T-t_{n}\right)^{-1}\left(-\log \left(T-t_{n}\right)\right)\right]^{\frac{1}{2}} \rightarrow 0,
$$

for a possibly more carefully chosen sequence $t_{n} \uparrow T$. However, we will see in this paper that $\lambda_{n}^{i}\left(T-t_{n}\right)^{-\frac{1}{2}-\varepsilon}$ will not converge to zero in general, however small we take $\varepsilon>0$.

Remark 1.8 Although we do not require it in this paper, it is not hard to improve (1.5) to, say,

$$
\left\|\tau\left(u\left(t_{n}\right)\right)\right\|_{L^{2}(D)}^{2}\left(T-t_{n}\right)\left(-\log \left(T-t_{n}\right)\right) \rightarrow 0 .
$$

Remark 1.9 In contrast to Theorem 1.4, we do not claim $L^{\infty}$ convergence in part (iii) of Theorem 1.6. Indeed, we will see in this paper that such convergence is simply false in general.

Remark 1.10 Theorem 1.6 holds for an arbitrary compact domain surface once the necessary notational changes have been made.

Remark 1.11 We must also record that bubbling is liable to occur at infinite time in addition to any bubbling at finite time. Indeed, it is easy to see from (1.4) and (1.3) that we may extract a sequence of times $t_{n} \rightarrow \infty$ so that $u\left(t_{n}\right): D \rightarrow \mathcal{N}$ satisfy the hypotheses of Theorem 1.4. An 'infinite-time singularity' is said to occur at any bubble point of this analysis.

The bubbling at infinite time raises the question of whether the convergence at times $t_{n}$ is in fact uniform as $t \rightarrow \infty$, and whether the energy concentrating at the bubble points can dissipate or move around the domain between consecutive times $t_{n}$ and $t_{n+1}$. For results and counterexamples along these lines see [14], [20], [16], and [18].

In contrast, because finite-time singular points are isolated in space-time, we are guaranteed reasonable uniform convergence of $u(t)$ away from bubble points, as $t$ tends to a singular time $T$, and concentrated energy cannot move dramatically around the domain between times $t_{n}$ and $t_{n+1}$ of Theorem 1.6. However, many other uniformity questions remain, many of which we settle here. Roughly speaking, it has been asked whether the images of the bubbles can move around in the target between times $t_{n}$ and $t_{n+1}$. This is popularly known as the question of 'uniqueness of bubbles.' For this to happen, the bubbles would have to move with unbounded speed as the singular time was approached.

We will see that such bubbles, moving in the image with unbounded speed can indeed occur. 
In order to continue a solution after a singularity, Struwe defines $u(T)$ to be the weak limit of $u\left(t_{n}\right)$ in $W^{1,2}$ as $n \rightarrow \infty$ and solves the harmonic map heat equation with $u(T)$ as initial data. Although $u(T) \in W^{1,2}$, a natural question which has been raised by Qing-Tian [12] and Lin-Wang [9] is whether $u(T)$ is continuous ${ }^{1}$. Indeed, this question is particularly relevant in the light of the results proved about infinite-time bubbling in [12] and [9].

We will see that $u(T)$ can in fact be discontinuous.

One explicit way of asking whether the flow converges well at the finite-time singularity is to lift the flow to the universal cover $\widehat{\mathcal{N}}$ of the target and see if the lifted bubbles still converge. More precisely, we make the following definition during which part (iv) of Theorem 1.6 should be kept in mind:

Definition 1.12 Given the regularity described in Theorem 1.1, we may lift a Struwe solution $u$ to a flow $\widehat{u}: D \times[0, \infty) \rightarrow \widehat{\mathcal{N}}$ into the universal cover $\widehat{\mathcal{N}}$ of the target manifold. We say that a singularity at a point $(x, T) \in D \times(0, \infty)$ of the flow $u$ is winding if there exist $\lambda_{n} \downarrow 0$, $a_{n} \rightarrow x, t_{n} \uparrow T$, points $\left\{x^{1}, \ldots, x^{k}\right\} \subset \mathbb{R}^{2}$ and a nonconstant harmonic map $\omega: \mathbb{R}^{2} \rightarrow \mathcal{N}$ such that $u\left(a_{n}+\lambda_{n} x, t_{n}\right) \rightarrow \omega(x)$ in $W_{l o c}^{2,2}\left(\mathbb{R}^{2} \backslash\left\{x^{1}, \ldots, x^{k}\right\}, \mathcal{N}\right)$ - and hence in $C_{\text {loc }}^{0}$ - but so that $\widehat{u}\left(a_{n}+\lambda_{n} x, t_{n}\right)$ does not converge in $C_{\text {loc }}^{0}\left(\mathbb{R}^{2} \backslash\left\{x^{1}, \ldots, x^{k}\right\}, \widehat{\mathcal{N}}\right)$ even after selecting a subsequence.

Remark 1.13 This definition makes sense for any compact target manifold $\mathcal{N}$, although if the target is simply connected then no singularity can be winding. The definition also makes sense for alternative domain surfaces, although if the domain is not simply connected then we only lift the flow in a neighbourhood of $(x, T)$ in space-time.

Roughly speaking then, a singularity is winding if at least one of the bubbles which develop must, once the flow has been lifted to $\widehat{\mathcal{N}}$, converge to infinity and escape every compact subdomain of $\widehat{\mathcal{N}}$.

We will see that winding singularities do indeed exist.

Next, we are interested in the rate of blow-up of finite-time singularities. With the selection of $t_{n}$ that we make in Theorem 1.6, we find in part (v) of that theorem that for any bubble,

$$
\lambda_{n}=o\left(\left(T-t_{n}\right)^{\frac{1}{2}}\right),
$$

and this may even be improved (see Remark 1.7) to

$$
\lambda_{n}=o\left(\left[\frac{\left(T-t_{n}\right)}{-\log \left(T-t_{n}\right)}\right]^{\frac{1}{2}}\right) .
$$

\footnotetext{
${ }^{1}$ See also the very recent paper of Qing [11] for a discussion about this question
} 
Lower bounds for $\lambda_{n}$ in terms of $T-t_{n}$ are much harder to obtain. Indeed, we do not know of any prior example of a finite-time singularity in the harmonic map heat flow for which any such lower bound has been obtained.

In this paper we have an example of a singularity for which for all $\delta>0$,

$$
\lambda_{n} \geq\left(T-t_{n}\right)^{\frac{1}{2}+\delta}
$$

for sufficiently large $n$.

The polynomial rate of blow-up is therefore precisely determined. We will, in fact, prove a marginally better bound (essentially writing $\delta$ above as a decaying function of $T-t_{n}$ ) and further marginal improvements to the rate could be achieved by altering the target metric carefully.

Finally, we return to the control on the tension provided by (1.4), and ask whether this can be improved.

We will see that although $\|\tau(u(t))\|_{L^{2}(D)}$ is in $L^{2}([0, T])$, it is not, in general, in $L^{2+\delta}([0, T])$ for any $\delta>0$.

We compile these results in the following main theorem, which is proved in Sections 3 and 4.

Theorem 1.14 There exist a compact target manifold $\mathcal{N}$ and an initial map $u_{0} \in C^{\infty}(D, \mathcal{N})$ such that the Struwe flow $u$ from Theorem 1.1 satisfies the following properties:

(a) There exists a singular point $(0, T) \in D \times(0, \infty)$ (with no singularity for $t<T$ );

(b) Precisely one bubble develops at $(0, T)$ in the sense that when analysed with Theorem 1.6, we must have $k=1$;

(c) The singularity is winding in the sense of Definition 1.12;

(d) The map $u(T) \in W^{1,2}(D, \mathcal{N})$ is discontinuous at the origin;

(e) For all $\delta>0$, the rate of blow-up of the bubble is constrained by

$$
\left(T-t_{n}\right)^{\frac{1}{2}+\delta} \ll \lambda_{n} \ll\left(T-t_{n}\right)^{\frac{1}{2}},
$$

where we write $a_{n} \ll b_{n}$ for positive sequences $\left\{a_{n}\right\}$ and $\left\{b_{n}\right\}$ if $\frac{a_{n}}{b_{n}} \rightarrow 0$ as $n \rightarrow \infty$.

(f) For all $\delta>0$, and $s \in[0, T)$, we have

$$
\int_{s}^{T}\|\tau(u(t))\|_{L^{2}(D)}^{2+\delta}=\infty .
$$

In the light of the work of L. Simon [14] it is tempting to conjecture that the nonuniformity of the bubbling that we witness in this work cannot occur if we insist on a real analytic target. 


\section{The bubbling analysis}

In this section we prove Theorem 1.6 based on the work of Struwe [15], Ding-Tian [4] and Qing-Tian [12] compiled in Theorem 1.4.

Our first task is to select the times $t_{n} \uparrow T$. By combining (1.4) and the fact that

$$
\int_{s}^{T} \frac{d t}{T-t}=\infty
$$

for $s<T$, we see that it is easy to pick times $t_{n} \uparrow T$ such that (1.5) is satisfied. The final sequence will be a subsequence of that picked now.

We now wish to analyse the flow at one of the singularities which occur at time $T$. This is a local analysis and we assume that the singularity occurs at the origin in $D$ to simplify the notation without losing any generality. Let $\nu>0$ be sufficiently small so that $\overline{D_{\nu}} \times\{T\}$ contains no other singular point. By the properties of $u$ from Theorem 1.1, we know that $u(t) \rightarrow u(T)$ in $C_{\text {loc }}^{k}\left(D_{\nu} \backslash\{0\}, \mathcal{N}\right)$, and we will use this fact implicitly in the following argument.

Let us look at the evolution of energy near 0. (These calculations should be compared to those of Qing [10].) After picking a cut-off function $\phi \in C^{\infty}([0, \infty),[0,1])$ supported in $[0,4)$, identically equal to 1 on $[0,1]$, and with $\left\|\phi^{\prime}\right\|_{L^{\infty}} \leq 1$, we may define, for $t \in[0, T]$ and $r \in\left(0, \frac{\nu}{2}\right]$, the cut energy

$$
\Theta_{r}(t):=\frac{1}{2} \int_{D} \phi^{2}\left(\frac{|y|^{2}}{r^{2}}\right)|\nabla u(t)|^{2}(y) d y .
$$

Differentiating with respect to $t$, using the equation (1.2), integrating by parts and using (1.1) (c.f. [16]) gives

$$
\frac{d \Theta_{r}(t)}{d t}=\int_{D} \phi^{2}\left(\frac{|y|^{2}}{r^{2}}\right) \nabla u . \nabla \tau=-\int_{D} \phi^{2}\left(\frac{|y|^{2}}{r^{2}}\right)|\tau|^{2}-\int_{D} 2 \phi\left(\frac{|y|^{2}}{r^{2}}\right) \phi^{\prime}\left(\frac{|y|^{2}}{r^{2}}\right) \frac{2}{r^{2}}[(y . \nabla) u] . \tau,
$$

and hence the estimate

$$
\left|\frac{d \Theta_{r}(t)}{d t}\right| \leq \int_{D}|\tau|^{2}+\frac{C}{r} \int_{D}\left|\nabla u\|\tau \mid \leq\| \tau(u(t))\left\|_{L^{2}(D)}^{2}+\frac{C}{r}\right\| \tau(u(t)) \|_{L^{2}(D)}\right.
$$

where $C$ is finally allowed to depend on $E\left(u_{0}\right)$. This expression may now be integrated between $a$ and $b$ where $0<a<b<T$ to give

$$
\begin{aligned}
\left|\Theta_{r}(b)-\Theta_{r}(a)\right| & \leq \int_{a}^{b}\|\tau(u(t))\|_{L^{2}(D)}^{2} d t+\frac{C}{r} \int_{a}^{b}\|\tau(u(t))\|_{L^{2}(D)} d t \\
& \leq \int_{a}^{T}\|\tau(u(t))\|_{L^{2}(D)}^{2} d t+\frac{C(T-a)^{\frac{1}{2}}}{r}\left(\int_{a}^{T}\|\tau(u(t))\|_{L^{2}(D)}^{2} d t\right)^{\frac{1}{2}} .
\end{aligned}
$$

Keeping in mind (1.4) we see that the right-hand side of (2.4) converges to zero as $a \uparrow T$, and hence that $\Theta_{r}(t)$ must have a limit as $t \uparrow T$. This allows us to define

$$
L:=\lim _{t \uparrow T} \Theta_{r}(t)-\Theta_{r}(T),
$$


where $L$ is not dependent on $r$, or equivalently

$$
\lim _{t \uparrow T} E\left(u(t), D_{r}\right)=L+E\left(u(T), D_{r}\right),
$$

(where we are using the $C^{k}$ convergence of $u(t)$ to $u(T)$ away from the origin). Referring back to Theorem 1.1 we then must have

$$
L=\lim _{r \downarrow 0} \lim _{t \uparrow T} E\left(u(t), D_{r}\right)>0 .
$$

Next we return to (2.4) setting $r=\gamma(T-a)^{\frac{1}{2}}$ (for some constant $\gamma>0$ ) and taking $b \uparrow T$ (keeping (2.5) in mind) to give

$$
\left|L+\Theta_{\gamma(T-a)^{\frac{1}{2}}}(T)-\Theta_{\gamma(T-a)^{\frac{1}{2}}}(a)\right| \leq \int_{a}^{T}\|\tau(u(t))\|_{L^{2}(D)}^{2} d t+\frac{C}{\gamma}\left(\int_{a}^{T}\|\tau(u(t))\|_{L^{2}(D)}^{2} d t\right)^{\frac{1}{2}} .
$$

Taking also the limit $a \uparrow T$ and using (1.4) again, we then see that

$$
\lim _{a \uparrow T} \Theta_{\gamma(T-a)^{\frac{1}{2}}}(a)=L,
$$

for all $\gamma>0$. Now, with $\alpha>0$, we may send $a \uparrow T$ in the inequality

$$
\Theta_{\frac{\alpha(T-a)^{\frac{1}{2}}}{2}}(a) \leq E\left(u(a), D_{\alpha(T-a)^{\frac{1}{2}}}\right) \leq \Theta_{\alpha(T-a)^{\frac{1}{2}}}(a) .
$$

Using (2.9) with $\gamma=\frac{\alpha}{2}$ and $\gamma=\alpha$, we find that

$$
\lim _{a \uparrow T} E\left(u(a), D_{\alpha(T-a)^{\frac{1}{2}}}\right)=L,
$$

for any $\alpha>0$.

At this point, let us take stock of which parts of the theorem we have addressed. In pursuit of part (ii) we have proved that

$$
\lim _{\eta \downarrow 0} \lim _{t \uparrow T} E\left(u(t), D_{\eta}\right)=\lim _{t \uparrow T} E\left(u(t), D_{(T-t)^{\frac{1}{2}}}\right)=L .
$$

Concerning part (iii), our estimates so far imply that

$$
\|u(t)-u(T)\|_{W^{1,2}\left(D_{\nu} \backslash D_{\alpha(T-t)} \frac{1}{2}\right)} \rightarrow 0,
$$

as $t \uparrow T$, for any $\alpha>0$.

It remains to analyse the bubbling which may occur at the singular point $(0, T) \in D \times(0, \infty)$ at the times $t_{n}$ selected earlier. For the remainder of the paper, we set $\mu_{n}:=\left(T-t_{n}\right)^{\frac{1}{2}}$. For this section, we define rescaled maps $u_{n}: D \rightarrow \mathcal{N}$ by $u_{n}(y)=u\left(\mu_{n} y, t_{n}\right)$.

By the scaling properties of the tension, and the already-established (1.5), we must have

$$
\left\|\tau\left(u_{n}\right)\right\|_{L^{2}(D)}=\mu_{n}\left\|\tau\left(u\left(t_{n}\right)\right)\right\|_{L^{2}\left(D_{\mu_{n}}\right)} \rightarrow 0
$$


and therefore we may apply Theorem 1.4 to the $u_{n}$. By $(2.10)$ we see that

$$
\lim _{n \rightarrow \infty} E\left(u_{n}, D_{\eta}\right)=L,
$$

for any $\eta \in(0,1]$. In particular,

$$
E\left(u_{n}, D \backslash D_{\eta}\right) \rightarrow 0
$$

as $n \rightarrow \infty$ for any $\eta \in(0,1]$ and so by part (a) of Theorem $1.4, u_{\infty}$ is forced to be constant on $D$.

It remains to make use of parts (i) to (iv) of Theorem 1.4. These find bubbles $\omega^{i}$, blow-up centres $a_{n}^{i}$ and bubble scales $\lambda_{n}^{i} \downarrow 0$. By (2.11), the origin is the only bubble point of $u_{n}$ (so $a_{n}^{i} \rightarrow 0$ as $n \rightarrow \infty)$. Scaling the blow-up centres and bubble scales by a factor $\mu_{n}$, then gives exactly those required for Theorem 1.6.

\section{The main construction}

In this section, we construct the target manifold and flow demanded by Theorem 1.14. Parts (a) to (c) of that theorem will be established here, with the final parts (d) to (f) left until Section 4 . The flow we construct will have various symmetries which will help us to establish the finer properties of Section 4. However, the construction itself is quite robust, and will guarantee not only a finite-time singularity but one that is winding, for much more general asymmetric initial maps.

In the sequel, we will concentrate on the flow up to the time of its first singularity. Whilst the flow is smooth, it will mainly suit us to consider the flow in terms of intrinsic coordinates on $\mathcal{N}$, ignoring the embedding $\mathcal{N} \hookrightarrow \mathbb{R}^{N}$ which is so useful in the earlier theory.

\subsection{Construction of the target manifold}

The target that we will construct will be a warped product manifold. Such manifolds have been useful in the construction of various examples of harmonic map flows - see [16], [17], and [20] and also of nonuniform behaviour in other geometric problems such as nonunique tangent maps for harmonic maps (see White [22]). Topologically, the target will be $[-1,1] \times S^{1} \times S^{2}$. Although this has nonempty boundary, we will later easily see that the flow remains within the smaller region $[0,1) \times S^{1} \times S^{2}$, so we may agree now to implicitly join the two boundary components to give $T^{2} \times S^{2}$ and modify the metric on the region $\left[-1,-\frac{1}{2}\right) \times S^{1} \times S^{2}$, say, to make it everywhere smooth. There is plenty of flexibility here, and in particular, we could cap each boundary component with a $D \times S^{2}$ to give $S^{2} \times S^{2}$, although no singularity could then be winding in the sense defined above.

Let us start off with the strip $\mathcal{S}=[-1,1] \times \mathbb{R}$, addressed by real coordinates $(w, z)$, and equipped with the metric

$$
\gamma=\left(\begin{array}{cc}
1 & w^{2} \\
w^{2} & 1+w^{4}
\end{array}\right)
$$


We then obtain a cylinder $\mathcal{C}$ by taking the quotient of $\mathcal{S}$ by the group of isometries $\Gamma=\{(w, z) \rightarrow$ $(w, z+n) \mid n \in \mathbb{Z}\}$.

Ultimately, we will only be concerned with the half of $\mathcal{C}$ where $w \geq 0$. On $\mathcal{S} \cap\{w>0\}$, we will mainly use coordinates $(x, y)$ where $x=\frac{1}{w}-z$ and $y=z$. (Equivalently $w=\frac{1}{x+y}$ and $z=y$.) We then have the restriction $x+y \geq 1$.

In these alternative coordinates, the group of isometries $\Gamma$ is now $\{(x, y) \rightarrow(x-n, y+n) \mid n \in \mathbb{Z}\}$, and the metric takes the form

$$
g=\left(\begin{array}{cc}
\frac{1}{(x+y)^{4}} & 0 \\
0 & 1
\end{array}\right),
$$

and we see that the curve $\{x=0\}$ on $\mathcal{C}$ (which should be visualised in $w, z$ coordinates, spiralling towards the circle $\{w=0\})$ is a geodesic.

We next define a warping function $f \in C^{\infty}(\mathcal{C})$ by

$$
f(w, z)= \begin{cases}e^{-\frac{2 \pi}{w}}\left(\sin 2 \pi\left(\frac{1}{w}-z-\frac{1}{8}\right)+\sqrt{2}\right)+1 & w>0 \\ 1 & w \leq 0 .\end{cases}
$$

(Note that $f$ is well defined on $\mathcal{C}$ despite being written in coordinates on $\mathcal{S}$.) More useful to us is the expression for $f$ written in $(x, y)$ coordinates on the half of $\mathcal{C}$ where $w>0$,

$$
f(x, y)=e^{-2 \pi(x+y)}\left(\sin 2 \pi\left(x-\frac{1}{8}\right)+\sqrt{2}\right)+1 .
$$

We note the following properties of $f$ :

(i) $f(w, z)>1$ for $w>0$, and $f(w, z)=1$ for $w \leq 0$,

(ii) $\frac{\partial f}{\partial y}=-2 \pi e^{-2 \pi(x+y)}\left(\sin 2 \pi\left(x-\frac{1}{8}\right)+\sqrt{2}\right)<0$ for $w>0$,

(iii) $\frac{\partial f}{\partial x}=e^{-2 \pi(x+y)}\left(-2 \pi \sin 2 \pi\left(x-\frac{1}{8}\right)-2 \sqrt{2} \pi+2 \pi \cos 2 \pi\left(x-\frac{1}{8}\right)\right)=2 \sqrt{2} \pi e^{-2 \pi(x+y)}(\cos 2 \pi x-1)$.

By property (iii) we have

$$
\frac{\partial f}{\partial x}(0, \cdot) \equiv 0
$$

and by (ii),

$$
\frac{\partial f}{\partial y}(0, y)=-\sqrt{2} \pi e^{-2 \pi y}
$$

Finally we define the target manifold to be the warped product

$$
\mathcal{N}=\mathcal{C} \times_{f} S^{2} .
$$

We denote the projections of $\mathcal{N}$ onto its two 2-dimensional components by $P_{1}: \mathcal{N} \rightarrow \mathcal{C}$ and $P_{1}: \mathcal{N} \rightarrow S^{2}$ respectively. 
The significance of (3.1) is that it may now be checked that the three dimensional noncompact manifold

$$
\Sigma:=\{(0, y) \mid y \geq 1\} \times S^{2}
$$

lies totally geodesically within $\mathcal{N}$.

\subsection{Analysis of the flow}

\subsubsection{Description of the flow and the flow equations}

In order to discuss flows into the target manifold $\mathcal{N}$ constructed in the previous section, we equip the $S^{2}$ part of $\mathcal{N}$ with coordinates. We choose spherical polar coordinates $(\alpha, \theta)$ where $\theta \in[0,2 \pi)$ is the longitude and $\alpha \in[0, \pi]$ gives a measure of latitude with $\alpha=0$ the 'north' pole and $\alpha=\pi$ the 'south' pole.

Let us now define an initial map $u_{0} \in C^{\infty}(D, \mathcal{N})$ for the harmonic map heat flow. Using polar coordinates $(r, \theta)$ on the domain, let

$$
u_{0}(r, \theta)=\left(0, y_{0}, \alpha_{0}(r), \theta\right),
$$

where the number $y_{0}>1$ and the function $\alpha_{0}:[0,1] \rightarrow \mathbb{R}$ with $\alpha_{0}(0)=0$ and $\alpha(1)=\pi$, will be fixed later to make $u_{0}$ smooth and of controlled energy. Therefore when projected onto $\mathcal{C}, u_{0}$ is constant, and when projected onto the $S^{2}, u_{0}$ maps once over the whole sphere. The map $u_{0}$ has constant boundary values.

Let us now take the usual Struwe solution of the harmonic map heat equation (as found by Theorem 1.1). For a certain nontrivial time interval $[0, T)$ (for some $T \in(0, \infty]$ ) we can be sure that the flow is both smooth, and remains within the interior of $\mathcal{C} \times S^{2}$. Moreover, during this time, by appealing to the uniqueness described in Theorem 1.1 the flow must remain within the totally geodesic submanifold $\Sigma$ described in (3.3) and must retain the rotational and corotational symmetry enjoyed by the initial map. We see, therefore, that the flow in these coordinates must take the form

$$
u(r, \theta, t)=(0, Y(r, t), \alpha(r, t), \theta),
$$

with boundary values $\frac{\partial Y}{\partial r}(0, \cdot) \equiv 0, Y(1, \cdot) \equiv y_{0}, \alpha(0, \cdot) \equiv 0$ and $\alpha(1, \cdot) \equiv \pi$, for $t \in[0, T)$.

Remark 3.1 Throughout this paper we will abuse notation as and when convenient by using $Y$ both as the function $Y(r, t)$ on $[0,1] \times[0, T)$ and as the corresponding rotationally symmetric function on $D \times[0, T)$ given by $(r, \theta, t) \rightarrow Y(r, t)$, and similarly for $\alpha$ etc.

It will be convenient to use the shorthand

$$
e(\alpha):=\frac{1}{2}\left[\left(\frac{\partial \alpha}{\partial r}\right)^{2}+\frac{\sin ^{2} \alpha}{r^{2}}\right] .
$$


The energy of $u(t)$ may then be written in terms of $Y$ and $\alpha$ as

$$
\begin{aligned}
E(t) & =\pi \int_{0}^{1}\left(\left(\frac{\partial Y}{\partial r}(r, t)\right)^{2} r+2 f(0, Y(r, t))[e(\alpha)(r, t)] r\right) d r . \\
& =\int_{D}\left(\frac{1}{2}|\nabla Y|^{2}+f(0, Y) e(\alpha)\right)
\end{aligned}
$$

Working either from this expression for the energy, or by working first with general asymmetric flows into $\mathcal{N}$ and then specialising to symmetric flows as considered here, we find (for $t \in[0, T)$ ) that the functions $Y$ and $\alpha$ solve the following PDE:

$$
\begin{gathered}
\frac{\partial Y}{\partial t}=\frac{\partial^{2} Y}{\partial r^{2}}+\frac{1}{r} \frac{\partial Y}{\partial r}-\frac{\partial f}{\partial y}(0, Y) e(\alpha) \\
\equiv \Delta Y-\frac{\partial f}{\partial y}(0, Y) e(\alpha) \\
\frac{\partial \alpha}{\partial t}=\frac{\partial^{2} \alpha}{\partial r^{2}}+\frac{1}{r} \frac{\partial \alpha}{\partial r}-\frac{\sin \alpha \cos \alpha}{r^{2}}+\frac{1}{f(0, Y)} \frac{\partial f}{\partial y}(0, Y) \frac{\partial Y}{\partial r} \frac{\partial \alpha}{\partial r}
\end{gathered}
$$

Having equation (3.8), and keeping in mind the fact that $\frac{\partial f}{\partial y}<0$, we may immediately apply the maximum principle to find that

$$
Y(r, t) \geq y_{0}>1
$$

for all $r \in[0,1]$ and $t \in[0, T)$. In particular, the flow cannot approach the boundary of $\mathcal{C} \times S^{2}$, and so without loss of generality, we may take $T \in(0, \infty]$ to be the largest possible value subject to the flow being smooth for times $t \in[0, T)$.

\subsubsection{Blow-up of $Y$}

We now try to analyse the fate of $Y$. We will see that $Y(0, t)$ is unbounded over some finite time interval; this corresponds to the development of a bubble at the centre of the domain disc in finite time. Moreover, once we have visualised how the submanifold $\Sigma$ from (3.3) sits within the target $\mathcal{N}$, we will see that the flow will then have the basic winding behaviour which we seek, and more specifically that the singularity will be winding in the sense of Definition 1.12.

First, we note that the flow cannot stay smooth, with uniform bounds on its derivatives, for all time. This is because the initial map $u_{0}$ has constant boundary values but is homotopically nontrivial. Any flow with this initial map which blew up neither at finite nor at infinite time, would provide a homotopy from $u_{0}$ to a harmonic map within the same nontrivial homotopy class. However, this is impossible since no such harmonic map exists by Theorem 1.2 of Lemaire.

According to our earlier notation, $T \in(0, \infty]$ is then the first blow-up time. By Theorem 1.6 (in the case $T<\infty$ ) or Remark 1.11 (in the case $T=\infty$ ) one or more bubbles $\omega: S^{2} \rightarrow \mathcal{N}$ must develop at time $t=T$; by the symmetry of the flow, and the finiteness of the number of singularities, the 
one and only bubble point must be the centre of the domain disc. We claim, roughly speaking, that any bubble must occur at " $y=\infty . "$

Lemma 3.2 Let $\omega: S^{2} \rightarrow \mathcal{N}$ be any bubble which develops at time $t=T$ in the flow constructed. Then the image of $\omega$ must lie within the three dimensional submanifold $\Omega$ of $\mathcal{N}$ defined by the condition $w=0$ (which is topologically $S^{1} \times S^{2}$ ). Moreover, with $P_{1}$ and $P_{2}$ the projections defined in Section 3.1 we must have $P_{1} \circ \omega$ a constant map, and $P_{2} \circ \omega$ a harmonic map between 2-spheres.

In the proof below, $\lambda_{n}$ will be the bubble scale for $\omega$ (as in Theorem 1.6 for example). Given the rotational symmetry of the flow, we may assume that $a_{n}=0$ for all $n$ - i.e. that the bubble is obtained by blowing up about the origin for each $n$.

Proof. We have already established that for times $t \in[0, T)$, the image of the flow lies within the submanifold $\Sigma$ described in (3.3). By either part (iv) of Theorem 1.6, or a combination of part (iv) of Theorem 1.4 combined with Remark 1.11 (depending on whether $T<\infty$ or $T=\infty$ ) we then see that the image of $\omega$ must lie within the closure of $\Sigma$ in $\mathcal{N}$. Taking the closure of $\Sigma$ adds the submanifold $\Omega$ of the lemma.

Meanwhile, since $\omega$ is a smooth map from $S^{2}$, and there is no finite length path in $\Omega \cup \Sigma$ between any point in $\Sigma$ and any point in $\Omega$, the image of $\omega$ must lie either wholly within $\Sigma$, or wholly within $\Omega$.

Suppose that the image of $\omega$ lay wholly within $\Sigma$. If $P_{2} \circ \omega$ were constant, then $P_{1} \circ \omega$ would be harmonic, and would take values in the geodesic $\{x=0\}$ within $\mathcal{C}$. Essentially $\omega$ would then be a harmonic function on the 2-sphere, and would therefore be constant, which cannot be the case for a bubble. Therefore, if the image of $\omega$ were to lie wholly within $\Sigma$, then $P_{2} \circ \omega$ would have to be nonconstant. In this case, if we define, for $s \in \mathbb{R}$ near 0 , the transformations $\phi_{s}: \mathcal{N} \rightarrow \mathcal{N}$ by

$$
\phi_{s}(x, y, \alpha, \theta)=(x, y+s, \alpha, \theta)
$$

then it is easy to check, using the inequality $\frac{\partial f}{\partial y}<0$, that

$$
\left.\frac{d}{d s}\right|_{s=0} E\left(\phi_{s} \circ \omega\right)<0,
$$

contradicting the fact that $\omega$ is harmonic.

We have established that the image of $\omega$ lies wholly within $\Omega$. But $\Omega$ is isometric to the cartesian product of a (scaled) $S^{1}$ and an $S^{2}$, and therefore $\omega$ splits into two harmonic maps $P_{1} \circ \omega: S^{2} \rightarrow$ $S^{1} \hookrightarrow \mathcal{C}$ and $P_{2} \circ \omega: S^{2} \rightarrow S^{2}$. Finally, any harmonic map from $S^{2}$ to $S^{1}$ can be lifted to a harmonic function, and must therefore be constant.

Having determined accurately the type of bubble which must occur, we are now in a position to prove that $T<\infty$ - i.e. that the first singularity must occur at finite time. Indeed, if the flow did 
not blow up at finite time, then we could apply Lemaire's Theorem 1.2 to deduce that the limit $u_{\infty}$ of the $u\left(t_{n}\right)$ from Remark 1.11 and Theorem 1.4 must be the constant map $u_{\infty}(r, \theta)=\left(0, y_{0}, \pi, \theta\right)$, and therefore by Remark 1.5, the image of at least one bubble would have to pass through the point $\left(0, y_{0}, \pi, \theta\right) \in \mathcal{N}$ which is impossible by Lemma 3.2.

Now we know that $T<\infty$, let $t_{n}$ and $k$ be as in the analysis of Theorem 1.6. We claim that $k=1$, which means that only one bubble can develop. Indeed, by the description of the bubbles given in Lemma 3.2, we see that any bubble has as much energy as a harmonic map between 2-spheres, which must have energy at least $4 \pi$ (see $[5,(11.5)]$ ). By choosing $y_{0}>1$ sufficiently large (to make $f\left(0, y_{0}\right)$ sufficiently close to 1$)$ and choosing $\alpha_{0}$ appropriately we are able to make the energy of $u_{0}$ as close as we like to $4 \pi$ (for example we may start by taking $\alpha_{0}=2 \tan ^{-1}(r / \varepsilon)$ with $\varepsilon>0$ small and perturb it slightly in order to fit the boundary condition $\alpha_{0}(1)=\pi$ ) with $u_{0}$ smooth. In particular we can make the energy strictly less than $8 \pi$, which then limits the flow to develop a single bubble. (We are implicitly using part (ii) of Theorem 1.6.) One implication of having only one bubble is that part (iv) of Theorem 1.6 applied to this singularity reduces to $u\left(\lambda_{n} \xi, t_{n}\right) \rightarrow \omega(\xi)$ as functions of $\xi$, in $W_{l o c}^{2,2}\left(\mathbb{R}^{2}, \mathcal{N}\right)$.

Finally, knowledge of the type of bubble which must occur also constrains $Y$ to have a particular type of blow-up at time $t=T$. In particular, the singularity must be winding (in the sense of Definition 1.12) since otherwise, after passing to a subsequence, not only would we have convergence of $u\left(\lambda_{n} \xi, t_{n}\right)$ in $W_{\text {loc }}^{2,2}\left(\mathbb{R}^{2}, \mathcal{N}\right)$, we would also have convergence of $\widehat{u}\left(\lambda_{n} \xi, t_{n}\right)$ in $C_{\text {loc }}^{0}\left(\mathbb{R}^{2}, \widehat{\mathcal{N}}\right)$ and hence convergence of $Y\left(\lambda_{n} \xi, t_{n}\right)$ in $C_{\text {loc }}^{0}\left(\mathbb{R}^{2}, \mathbb{R}\right)$. However, by considering again how $\Sigma$ and $\Omega$ sit within $\mathcal{N}$, we see that the only way that $u\left(\lambda_{n} \xi, t_{n}\right)$ can converge to a bubble of the type described in Lemma 3.2 is if $Y\left(\lambda_{n} \xi, t_{n}\right) \rightarrow \infty$ for every $\xi \in \mathbb{R}^{2}$, which is contradictory.

In conclusion, we have established that a singularity develops in the flow at the origin, at finite time. Precisely one bubble is created, and the bubble is winding in the sense of Definition 1.12.

\section{Finer properties of the singularity}

We now wish to finish the proof of Theorem 1.14 by addressing parts (d) to (f). We continue to switch between notations for $Y$ as warned in Remark 3.1.

\subsection{Oscillation of $Y$}

In this section we establish decay of the oscillation of $Y\left(t_{n}\right)$ over scales of length $\mu_{n}:=\left(T-t_{n}\right)^{\frac{1}{2}}$. We define the oscillation of a function or map $f$ over a region $\Omega$ by

$$
\operatorname{osc}(f, \Omega):=\sup _{a, b \in \Omega} \operatorname{dist}(f(a), f(b)) .
$$


Lemma 4.1 The flow constructed in Section 3.2.1 satisfies

$$
\operatorname{osc}\left(Y\left(t_{n}\right), D_{\mu_{n}}\right) \rightarrow 0
$$

as $n \rightarrow \infty$.

Remark 4.2 For general flows, from arbitrary compact domain surfaces into arbitrary compact target manifolds, there is a weaker analogue of Lemma 4.1 which states that after lifting the flow around a neighbourhood of a singularity to the universal cover of the target, the oscillation of the lifted flow $\widehat{u}$ over $D_{\mu_{n}}$ at time $t_{n}$, is bounded uniformly in $n$. This weaker result would in fact suffice for our requirements in this paper.

Proof. In Section 3.2.2 we established that precisely one bubble developed in the sequence $u\left(t_{n}\right)$, at a scale $\lambda_{n}$, say. Moreover, by Lemma 3.2, and part (iv) of Theorem 1.6, we know that $P_{1} \circ u\left(\lambda_{n} \xi, t_{n}\right) \rightarrow p$ as functions of $\xi$, in $C_{\text {loc }}^{0}\left(\mathbb{R}^{2}, \mathcal{C}\right)$ where $p:=$ image $\left(P_{1} \circ \omega\right) \in \mathcal{C}$. In particular, we have

$$
\operatorname{osc}\left(Y\left(t_{n}\right), D_{R \lambda_{n}}\right) \rightarrow 0
$$

for any $R \in(0, \infty)$, as $n \rightarrow \infty$.

We now define rescaled maps $u_{n}: D \rightarrow \mathcal{N}$ by $u_{n}(\xi):=u\left(2 \mu_{n} \xi, t_{n}\right)$. (We may assume that $\mu_{n} \leq \frac{1}{2}$ for all $n$.) In this new language, by appealing to Theorem 1.6 (parts (v) and (iii)) we see that $u_{n}$ develops the same bubble at a scale $\lambda_{n}\left(2 \mu_{n}\right)^{-1} \rightarrow 0$, and that $u_{n}$ converges to a constant map in $W_{l o c}^{1,2}(D \backslash\{0\}, \mathcal{N})$. (Note once again that no bubble develops at the scale $\mu_{n}$ in the maps $u\left(t_{n}\right)$.) By the scaling properties of the tension, and by (1.5) of Theorem 1.6 we have

$$
\left\|\tau\left(u_{n}\right)\right\|_{L^{2}(D)}=\mu_{n}\left\|\tau\left(u\left(t_{n}\right)\right)\right\|_{L^{2}\left(D_{2 \mu_{n}}\right)} \rightarrow 0,
$$

as $n \rightarrow \infty$. We are then in a position to apply Theorem 1.4 to the $u_{n}$. Part (b) of that theorem tells us that $u_{n}$ converges to a constant not only in $W_{l o c}^{1,2}(D \backslash\{0\}, \mathcal{N})$ but also in $L_{l o c}^{\infty}(D \backslash\{0\}, \mathcal{N})$. In particular, we must have

$$
\operatorname{osc}\left(Y\left(t_{n}\right), D_{\mu_{n}} \backslash D_{2 \nu \mu_{n}}\right) \rightarrow 0
$$

for arbitrarily small $\nu>0$, as $n \rightarrow \infty$.

We also gain the important $L^{\infty}$ convergence of part (iii) of Theorem 1.4 (which is the contribution of Qing-Tian [12]). This implies that for arbitrarily small $\varepsilon>0$, there exist $R>0$ and $\nu \in(0,1]$ such that we have the 'neck estimate'

$$
\operatorname{Osc}\left(u_{n}, D_{\nu} \backslash D_{R \lambda_{n}\left(2 \mu_{n}\right)^{-1}}\right)<\varepsilon
$$

for sufficiently large $n$. Renormalising, we have

$$
\operatorname{osc}\left(Y\left(t_{n}\right), D_{2 \mu_{n} \nu} \backslash D_{R \lambda_{n}}\right)<\varepsilon
$$

for sufficiently large $n$. Combining (4.1), (4.2) and (4.3) then gives us the lemma. 


\subsection{Discontinuity of $u(T)$}

In this section we prove that $Y(T)$, the weak limit of $Y\left(t_{n}\right)$ in $W^{1,2}(D, \mathbb{R})$, must be discontinuous, and hence that $u(T)$ must also be discontinuous as claimed in part (d) of Theorem 1.14. (It is important to keep in mind that the image of the flow for $t \in[0, T)$ remains in $\Sigma$, and that the flow $u$ is smooth away from the origin, up to and including time $t=T$.) The function $Y(T)$ must be smooth away from the origin. Our aim is to prove that it must be unbounded.

We begin by defining a measure of the average of $Y$ over certain annuli in $D$. Let $\phi \in C^{\infty}([0, \infty),[0,1])$ be nonconstant and be supported in the interval $\left[\frac{1}{4}, 1\right]$. We may then define, for $r \in(0,1]$ and $t \in[0, T]$,

$$
\Psi_{r}(t):=\int_{D} \frac{1}{r^{2}} \phi\left(\frac{|\xi|^{2}}{r^{2}}\right) Y(\xi, t) d \xi
$$

Retaining the notation $\mu_{n}:=\left(T-t_{n}\right)^{\frac{1}{2}}$ from before, we then consider $\Psi_{\mu_{n}}\left(t_{n}\right)$. We established during Section 3.2.2 that $Y\left(0, t_{n}\right) \rightarrow \infty$. Combining with Lemma 4.1, we see that $\inf _{\xi \in D} Y\left(\mu_{n} \xi, t_{n}\right) \rightarrow$ $\infty$. In particular, this forces

$$
\Psi_{\mu_{n}}\left(t_{n}\right) \rightarrow \infty
$$

as $n \rightarrow \infty$. We now try to control how $\Psi_{r}$ evolves over short time intervals. Differentiating, and using 3.8 , we find that

$$
\begin{aligned}
\frac{d \Psi_{r}}{d t} & =\int_{D} \frac{1}{r^{2}} \phi\left(\frac{|\xi|^{2}}{r^{2}}\right) \frac{\partial Y}{\partial t}(\xi, t) d \xi \\
& =\int_{D} \frac{1}{r^{2}} \phi\left(\frac{|\xi|^{2}}{r^{2}}\right)\left[\Delta Y-\frac{\partial f}{\partial y}(0, Y) e(\alpha)\right] \\
& =\int_{D}\left[\frac{1}{r^{2}} \phi\left(\frac{|\xi|^{2}}{r^{2}}\right)\left(-\frac{\partial f}{\partial y}(0, Y)\right) e(\alpha)-\frac{1}{r^{2}} \phi^{\prime}\left(\frac{|\xi|^{2}}{r^{2}}\right) \frac{2}{r^{2}} \xi . \nabla Y\right]
\end{aligned}
$$

Keeping in mind the boundedness of the energy, written as in (3.6), the inequality $f \geq 1$, and the fact that by (3.2) and (3.10)

$$
-\frac{\partial f}{\partial y}(0, y) \leq \sqrt{2} \pi
$$

we may then estimate

$$
\left|\frac{d \Psi_{r}}{d t}\right| \leq \frac{C}{r^{2}} \int_{D} e(\alpha)+\frac{C}{r^{3}} \int_{D_{r}}|\nabla Y| \leq \frac{C}{r^{2}},
$$

where $C$ depends on the choice of $\phi$ and $E(0)$. Specialising to the case $r=\mu_{n}$ and integrating from time $t_{n}$ to some later time $t \in\left[t_{n}, T\right)$, we then find that

$$
\left|\Psi_{\mu_{n}}(t)-\Psi_{\mu_{n}}\left(t_{n}\right)\right| \leq\left(T-t_{n}\right) \sup \left|\frac{d \Psi_{\mu_{n}}}{d t}\right| \leq C
$$

with $C$ independent of $n$ and $t$. Keeping in mind (4.4), we must have

$$
\lim _{n \rightarrow \infty} \liminf _{t \uparrow T} \Psi_{\mu_{n}}(t) \rightarrow \infty .
$$


However, as mentioned earlier, if $u(T)$ were continuous, $Y(T)$ would be a bounded function. By smoothness of the flow on $D \backslash\{0\} \times(0, T]$ we would then have

$$
\lim _{t \uparrow T} \Psi_{\mu_{n}}(t)=\Psi_{\mu_{n}}(T) \leq M,
$$

for some $M<\infty$ independent of $n$, which would contradict (4.5). We conclude that $u(T)$ must be discontinuous as claimed.

\subsection{Upper bounds for $Y$}

Although we know from Section 3.2.2 that $Y\left(0, t_{n}\right) \rightarrow \infty$ as $n \rightarrow \infty$, we do not yet have any control on how fast $Y\left(\cdot, t_{n}\right)$ blows up in relation to $\left(T-t_{n}\right)^{-1}$.

Lemma 4.3 The flow constructed in Section 3.2.1 satisfies

$$
M_{n}:=\sup _{D} Y\left(\cdot, t_{n}\right) \leq C\left[-\ln \left(T-t_{n}\right)\right]^{\frac{1}{2}},
$$

with $C$ independent of $n$, for sufficiently large $n$.

Proof. The strategy behind the proof is that if $Y\left(0, t_{n}\right)$ were too large, then $Y\left(\cdot, t_{n}\right)$ would be large on a disc of radius $D_{\mu_{n}}$ by Lemma 4.1, and this would force the Dirichlet energy of $Y\left(t_{n}\right)$ to be unreasonably large.

In more detail, we may use (3.6) and the fact that $E(t)$ is a weakly decreasing function of $t \in[0, T)$ to estimate

$$
E(0) \geq E(t) \geq \frac{1}{2} \int_{D}|\nabla Y(t)|^{2} \geq \frac{1}{2} \int_{D \backslash D_{s}}|\nabla Y(t)|^{2},
$$

for $s \in(0,1)$. The final term, being the Dirichlet energy of $Y(t)$, will be at least the Dirichlet energy of the unique harmonic function with the same values as $Y(t)$ on the boundary $\partial\left(D \backslash D_{s}\right)$. That function is $(r, \theta) \rightarrow\left(\frac{\ln r}{\ln s}\right)\left(Y(s, t)-y_{0}\right)+y_{0}$, which has energy $\frac{\pi}{-\ln s}\left(Y(s, t)-y_{0}\right)^{2}$. Therefore, we find that for all $t \in[0, T)$ and $s \in(0,1)$,

$$
Y(s, t) \leq\left[\frac{E(0)}{\pi}(-\ln s)\right]^{\frac{1}{2}}+y_{0} .
$$

In particular, for all $s \in\left[\mu_{n}, 1\right]$, we have

$$
Y\left(s, t_{n}\right) \leq C\left[\left(-\ln \left(T-t_{n}\right)\right)\right]^{\frac{1}{2}},
$$

for sufficiently large $n$. This proves the desired estimate on the annulus $D \backslash D_{\mu_{n}}$, and may be combined with Lemma 4.1 to extend the estimate to the whole disc $D$. 


\subsection{Rate of blow-up}

In this section we prove the lower bound on the bubble scale $\lambda_{n}$ which is claimed in part (e) of Theorem 1.14 and states that for all $\delta>0, \lambda_{n}\left(T-t_{n}\right)^{-\left(\frac{1}{2}+\delta\right)} \rightarrow \infty$ as $n \rightarrow \infty$. (The upper bound of part (e) has already been proved for general flows as part $(\mathrm{v})$ of Theorem 1.6.)

Our strategy will be to argue that if the flow were too concentrated at time $t_{n}$, then the tension of $u\left(t_{n}\right)$ would be unreasonably large, in some sense. We will estimate the tension from below by constructing an explicit variation of $u\left(t_{n}\right)$ which will create a large drop in the energy. In the process, we will require a 'neck analysis' (see Lemma 4.4 below) and the control of the blow-up of $Y$ we established in Lemma 4.3. The first result about necks in the context of maps with small but nonzero tension was proved by Qing-Tian [12] and their analysis is strong enough for our purposes. Here we quote a slight simplification and perturbation of Lemma 2.9 of [18] (see also Remark 2.8 of [18]) the proof of which was more along the lines of Lin-Wang [9].

Lemma 4.4 Suppose that $v: D \rightarrow \mathcal{N}$ is smooth and satisfies $E(v, D)<M$ for some $M$. Then there exist $\delta>0$ (dependent only on $\mathcal{N}$ ) and $K>0$ (dependent only on $M$ and $\mathcal{N}$ ) such that if

$$
E\left(v, D \backslash D_{r^{2}}\right)<\delta
$$

for some $r \in\left(0, \frac{1}{2}\right]$, and

$$
\|\tau(v)\|_{L^{2}(D)}^{2}<\delta
$$

then we have the estimate

$$
E\left(v, D_{2 r} \backslash D_{r}\right) \leq K r
$$

Here, as in [18], this sort of lemma will allow us to argue that if the bubble scale is sufficiently small then we can find a dyadic annulus $D_{2 r} \backslash D_{r}$ surrounding the part of the domain where concentration is occurring, and with very small energy. Such energy control then allows us to analyse the concentrated part of the flow (which is converging to the bubble) in isolation from the rest of the flow.

As in previous sections we use the shorthand $\mu_{n}:=\left(T-t_{n}\right)^{\frac{1}{2}}$ and consider rescaled maps $u_{n}$ : $D \rightarrow \mathcal{N}$ defined this time by $u_{n}(\xi)=u\left(\mu_{n} \xi, t_{n}\right)$. As before, by (1.5) we have $\tau\left(u_{n}\right) \rightarrow 0$ in $L^{2}(D)$. Retaining the notation $\lambda_{n}$ for the scale of the (unique) bubble produced by Theorem 1.6 (and keeping in mind parts (v) and (iii) of that theorem) the rescaled maps $u_{n}$ must develop a bubble at the scale $\lambda_{n} / \mu_{n} \rightarrow 0$ and we must have

$$
\lim _{R \rightarrow \infty} \lim _{n \rightarrow \infty} E\left(u_{n}, D \backslash D_{\frac{R \lambda_{n}}{\mu_{n}}}\right)=0 .
$$

In particular, we may fix $R>0$ sufficiently large so that

$$
E\left(u_{n}, D \backslash D_{\frac{R \lambda_{n}}{\mu_{n}}}\right)<\delta
$$


for sufficiently large $n$ (where $\delta$ is as in Lemma 4.4). Therefore, after setting $r_{n}:=\sqrt{\frac{R \lambda_{n}}{\mu_{n}}}$, we may apply Lemma 4.4 for sufficiently large $n$ to deduce that

$$
E\left(u_{n}, D_{2 r_{n}} \backslash D_{r_{n}}\right) \leq K r_{n}
$$

Retranslating to the original scale gives

$$
E\left(u\left(t_{n}\right), A_{n}\right) \leq K r_{n}
$$

where $A_{n}:=D_{2 \sqrt{R \lambda_{n} \mu_{n}}} \backslash D_{\sqrt{R \lambda_{n} \mu_{n}}}$.

We now wish to describe explicitly a variation of the map $u\left(t_{n}\right)$ which will reduce its energy by a controlled amount, and thus give control on its tension. The variation will be written in terms of the $Y$ and $\alpha$ of Section 3.2.1, and so let us write $Y_{n}:=Y\left(\cdot, t_{n}\right)$ and $\alpha_{n}:=\alpha\left(\cdot, t_{n}\right)$. We define a cut-off function $\phi \in C^{\infty}([0, \infty),[0,1])$ supported in $[0,4)$, identically equal to 1 on $[0,1]$, and with $\left\|\phi^{\prime}\right\|_{L^{\infty}} \leq 1$, and a scaled cut-off $\phi_{n}: D \rightarrow \mathbb{R}$ by

$$
\phi_{n}(\xi)=\phi\left(\frac{|\xi|^{2}}{R \lambda_{n} \mu_{n}}\right)
$$

We may then vary $u\left(t_{n}\right)$ through a family of maps $v_{n}^{(s)}: D \rightarrow \mathcal{N}$ where, for $s$ in a neighbourhood of $0 \in \mathbb{R}$, we define (using the coordinate notation for $\mathcal{N}$ of Section 3.2.1)

$$
v_{n}^{(s)}(\xi)=\left(0, Y_{n}(\xi)+s \phi_{n}(\xi), \alpha_{n}(\xi), \theta\right),
$$

so that $v_{n}^{(0)} \equiv u\left(t_{n}\right)$.

Writing the energy in terms of these coordinates (c.f. (3.6)) gives

$$
E\left(v_{n}^{(s)}\right)=\int_{D}\left(\frac{1}{2}\left|\nabla\left(Y_{n}+s \phi_{n}\right)\right|^{2}+f\left(0, Y_{n}+s \phi_{n}\right) e\left(\alpha_{n}\right)\right)
$$

and hence

$$
\left.\frac{d}{d s} E\left(v_{n}^{(s)}\right)\right|_{s=0}=\int_{D} \nabla Y_{n} \cdot \nabla \phi_{n}+\int_{D} \frac{\partial f}{\partial y}\left(0, Y_{n}\right) \phi_{n} e\left(\alpha_{n}\right) .
$$

Let us label the two terms on the right-hand side $I$ and $I I$ respectively. First we estimate

$$
\begin{aligned}
|I| & =\left|\int_{A_{n}} \nabla Y_{n} \cdot \nabla \phi_{n}\right| \leq\left(\int_{A_{n}}\left|\nabla Y_{n}\right|^{2}\right)^{\frac{1}{2}}\left(\int_{A_{n}}\left|\nabla \phi_{n}\right|^{2}\right)^{\frac{1}{2}} \\
& \leq C\left(E\left(u\left(t_{n}\right), A_{n}\right)\right)^{\frac{1}{2}} \leq C(K) r_{n}^{\frac{1}{2}} \leq C(K, R)\left(\frac{\lambda_{n}}{\mu_{n}}\right)^{\frac{1}{4}}
\end{aligned}
$$

where we have used the fact that $\nabla \phi_{n}$ is supported on $A_{n}$, the Cauchy-Schwarz inequality, the dilation invariance of the energy, and (4.8).

Next we wish to estimate $I I$. By (3.2)

$$
-\frac{\partial f}{\partial y}\left(0, Y_{n}\right)=\sqrt{2} \pi e^{-2 \pi Y_{n}} \geq e^{-2 \pi Y_{n}} \geq e^{-2 \pi M_{n}}
$$


where we are still using the notation $M_{n}:=\sup _{D} Y_{n}$. By the description of the bubble $\omega$ given by Lemma 3.2, and the expression for the energy (3.6) we have

$$
\int_{D_{\lambda_{n}}} e\left(\alpha_{n}\right)=E\left(P_{2} \circ u\left(t_{n}\right), D_{\lambda_{n}}\right) \rightarrow E\left(P_{2} \circ \omega, D\right)=: \kappa>0,
$$

where $P_{2}$ is the projection $\mathcal{N} \rightarrow S^{2}$ as in Section 3.1. Moreover, we may use the facts that $\phi_{n} \equiv 1$ on $D_{\sqrt{R \lambda_{n} \mu_{n}}}$ and that $\frac{\lambda_{n}}{\mu_{n}} \rightarrow 0$ to deduce that

$$
\int_{D} \phi_{n} e\left(\alpha_{n}\right) \geq \int_{D_{\lambda_{n}}} e\left(\alpha_{n}\right)
$$

for sufficiently large $n$. Combining (4.12), (4.13) and (4.14), we have

$$
-I I \geq e^{-2 \pi M_{n}} \int_{D} \phi_{n} e\left(\alpha_{n}\right) \geq e^{-2 \pi M_{n}} \frac{\kappa}{2},
$$

for sufficiently large $n$.

Finally, we deal with the left-hand side of (4.9) by exploiting the fact that the tension is the $L^{2}$ gradient of the energy, and so, using suggestive notation,

$$
\begin{aligned}
\left|\frac{d}{d s} E\left(v_{n}^{(s)}\right)\right|_{s=0} \mid & =\left|\int_{D}\left\langle\tau\left(u\left(t_{n}\right)\right),\left.\frac{d}{d s} v_{n}^{(s)}\right|_{s=0}\right\rangle\right| \leq\left\|\tau\left(u\left(t_{n}\right)\right)\right\|_{L^{2}(D)}\left\|\phi_{n}\right\|_{L^{2}(D)} \\
& \leq C \sqrt{R \lambda_{n} \mu_{n}}\left\|\tau\left(u\left(t_{n}\right)\right)\right\|_{L^{2}(D)} \leq C(R) \sqrt{\frac{\lambda_{n}}{\mu_{n}}}
\end{aligned}
$$

for sufficiently large $n$, because of (1.5).

Combining our knowledge about all three terms of (4.9), we see that

$$
e^{-2 \pi M_{n}} \leq C(-I I) \leq C\left(\frac{\lambda_{n}}{\mu_{n}}\right)^{\frac{1}{4}}+C\left(\frac{\lambda_{n}}{\mu_{n}}\right)^{\frac{1}{2}} \leq C\left(\frac{\lambda_{n}}{\mu_{n}}\right)^{\frac{1}{4}}
$$

for sufficiently large $n$, with $C$ independent of $n$. Incorporating the control on $M_{n}$ offered by Lemma 4.3 , we then have

$$
\exp \left(-C\left[-\ln \left(T-t_{n}\right)\right]^{\frac{1}{2}}\right) \leq \frac{\lambda_{n}}{\mu_{n}},
$$

for sufficiently large $n$. Rewriting, we have

$$
\lambda_{n} \geq\left(T-t_{n}\right)^{\frac{1}{2}+C\left[-\ln \left(T-t_{n}\right)\right]^{-\frac{1}{2}},}
$$

which is stronger than the bound claimed in the theorem.

\subsection{Estimates on the tension}

By (1.4) we know that for any Struwe solution of the harmonic map heat equation (as in Theorem 1.1) we always have $\|\tau(u(t))\|_{L^{2}(D)} \in L^{2}([0, \infty))$ as a function of $t$. We now wish to point out that 
for the flow we have constructed in this paper, this cannot be improved in that

$$
\|\tau(u(t))\|_{L^{2}(D)} \notin L^{2+\varepsilon}((T-\varepsilon, T))
$$

for any $\varepsilon>0$, as claimed in part (f) of Theorem 1.14.

To establish this, we use the control on the rate of blow-up we found in Section 4.4 (which makes up part (e) of Theorem 1.14). Interpreting this in the language of the cut energy from Section 2, we see that

$$
\Theta_{\left(T-t_{n}\right)^{\frac{1}{2}+\delta}}\left(t_{n}\right) \rightarrow 0
$$

as $n \rightarrow \infty$. In contrast, with $L$ defined as in Section 2, we must have

$$
\lim _{t \uparrow T} \Theta_{\left(T-t_{n}\right)^{\frac{1}{2}+\delta}}(t) \geq L>0,
$$

for fixed $n$. Combining, we see, for sufficiently large $n$, that

$$
0<\frac{L}{2} \leq \lim _{t \uparrow T} \Theta_{\left(T-t_{n}\right)^{\frac{1}{2}+\delta}}(t)-\Theta_{\left(T-t_{n}\right)^{\frac{1}{2}+\delta}}\left(t_{n}\right)=\int_{t_{n}}^{T} \frac{d}{d t} \Theta_{\left(T-t_{n}\right)^{\frac{1}{2}+\delta}}(t) d t .
$$

We then return to (2.1) of Section 2 and note the variant of (2.2)

$$
\frac{d \Theta_{r}(t)}{d t} \leq \frac{C}{r}\|\tau(u(t))\|_{L^{2}(D)} .
$$

In our situation this gives, via the Hölder inequality,

$$
\frac{L}{2} \leq \frac{C}{\left(T-t_{n}\right)^{\frac{1}{2}+\delta}} \int_{t_{n}}^{T}\|\tau(u(t))\|_{L^{2}(D)} d t \leq\left(\int_{t_{n}}^{T}\|\tau(u(t))\|_{L^{2}(D)}^{\frac{2}{1-2 \delta}} d t\right)^{\frac{1}{2}-\delta} .
$$

In particular, for any $\varepsilon>0$, the quantity

$$
\int_{t_{n}}^{T}\|\tau(u(t))\|_{L^{2}(D)}^{2+\varepsilon} d t
$$

must be bounded below by a strictly positive number, independent of $n$, which implies our claim.

\section{Appendix}

We end by sketching a proof of Lemma 1.3. We may take the target $\mathcal{N}$ to be the warped product $S^{1} \times{ }_{f} S^{2}$ where $f: S^{1} \rightarrow[1,3]$ is defined to be $f(\theta)=2-\cos \theta$. Note now that since $\min f=f(0)=$ 1 , the least energy of a map $S^{2} \rightarrow \mathcal{N}$ which has degree one once projected onto the $S^{2}$ component of the target, is $4 \pi$. Fix now a point $q \in S^{2}$, and choose a sequence of smooth maps $v_{n}: D \rightarrow \mathcal{N}$ with the properties that $\left.v_{n}\right|_{\partial D}=\left(\frac{\pi}{2}, q\right)$, the projection of $v_{n}$ onto the $S^{2}$ component of the target covers the sphere precisely once, and $E\left(v_{n}\right) \downarrow 4 \pi$. (A typical such map sends a small disc in the domain conformally over most of $\{0\} \times S^{2}$ - costing a little less than $4 \pi$ of energy - and then uses the rest of the domain to match up with the boundary values - costing very little extra energy.) 
We now claim that the $v_{0}$ of the lemma may be taken to be one of the $v_{n}$ in this sequence. If that were not the case, then for all $n$, we could deform $v_{n}$ to a lower energy smooth map with arbitrarily small tension in $L^{2}$. In particular, we could find a new sequence of smooth maps $u_{n}: D \rightarrow \mathcal{N}$ with the properties that $\left.u_{n}\right|_{\partial D}=\left(\frac{\pi}{2}, q\right)$, the projection of $u_{n}$ onto the $S^{2}$ component of the target is still homotopically nontrivial, $E\left(u_{n}\right) \downarrow 4 \pi$, and $\tau\left(u_{n}\right) \rightarrow 0$ in $L^{2}(D)$ as $n \rightarrow \infty$.

Such a sequence of maps undergoes bubbling, as described in Theorem 1.4 of this paper. By Lemaire's Theorem 1.2, the map $u_{\infty}$ must send the whole disc to the point $\left(\frac{\pi}{2}, q\right)$. A more elaborate version of Theorem 1.4 in which we considered the possibility of bubbling at the boundary would tell us that a bubble must then be created, with energy $4 \pi$, and the only bubbles with this energy which can account for the change in homotopy class between $u_{n}$ and $u_{\infty}$ are harmonic maps $\omega: S^{2} \rightarrow \mathcal{N}$ with image $\{0\} \times S^{2}$. However, by Qing-Tian's 'no necks' result - see Remark 1.5 - extended suitably to cover singularities at the boundary, the image of $u_{\infty}$ cannot be disconnected from the image of $\omega$, so we have a contradiction.

\section{References}

[1] M. BERTSCH, R. DAL PASSO and R. VAN DER HOUT, Nonuniqueness for the heat flow of harmonic maps on the disk. Arch. Ration. Mech. Anal. 161 (2002) 93-112.

[2] K.-C. CHANG, Heat flow and boundary value problem for harmonic maps. Ann. Inst. Henri Poicaré, Analyse nonlinéaire 6 No. 5 (1989) 363-395.

[3] K.-C. CHANG, W.-Y. DING and R. YE, Finite-time Blow-up of the Heat Flow of Harmonic Maps from Surfaces. J. Diff. Geom. 36 (1992) 507-515.

[4] W.-Y. DING and G. TIAN, Energy identity for a class of approximate harmonic maps from surfaces. Comm. Anal. Geom. 3 (1995) 543-554.

[5] J. EELLS and L. LEMAIRE, A report on harmonic maps. Bull. London Math. Soc. 10 (1978) $1-68$.

[6] J. EELLS and J. H. SAMPSON, Harmonic mappings of Riemannian manifolds. Amer. J. Math. 86 (1964) 109-169.

[7] A. FREIRE, Uniqueness for the harmonic map flow from surfaces to general targets. Comment. Math. Helv. 70 (1995) 310-338; correction in Comment. Math. Helv. 71 (1996) 330-337.

[8] L. LEMAIRE, Applications harmoniques de surfaces riemannienes. J. Diff. Geom. 13 (1978) 51-78.

[9] F.-H. LIN and C.-Y. WANG, Energy identity of harmonic map flows from surfaces at finite singular time. Calc. Var. Partial Differential Equations 6 (1998) 369-380.

[10] J. QING, On singularities of the heat flow for harmonic maps from surfaces into spheres. Comm. Anal. Geom. 3 (1995) 297-315.

[11] J. QING, A remark on the finite time singularity of the heat flow for harmonic maps. to appear, Calc. Var. 
[12] J. QING and G. TIAN, Bubbling of the heat flows for harmonic maps from surfaces. Comm. Pure Appl. Math. 50 (1997) 295-310.

[13] J. SACKS and K. UHLENBECK, The existence of minimal immersions of 2-spheres. Ann. Math. 113 (1981) 1-24.

[14] L. SIMON, Asymptotics for a class of nonlinear evolution equations, with applications to geometric problems. Ann. Math. 118 (1983) 525-571.

[15] M. STRUWE, On the evolution of harmonic mappings of Riemannian surfaces. Comment. Math. Helv. 60 (1985) 558-581.

[16] P. M. TOPPING, Rigidity in the Harmonic Map Heat Flow. J. Differential Geometry 45 (1997) 593-610.

[17] P. M. TOPPING, An example of a nontrivial bubble tree in the harmonic map heat flow. Harmonic morphisms, harmonic maps and related topics (Brest, 1997) CRC Res. Notes Math., 413 (2000) 185-191.

[18] P. M. TOPPING, Repulsion and quantization in almost-harmonic maps, and asymptotics of the harmonic map flow. to appear, Annals of Math.

[19] P. M. TOPPING, Reverse bubbling and nonuniqueness in the harmonic map flow. Int. Math. Res. Not. 10 (2002) 505-520.

[20] P. M. TOPPING, The Harmonic Map Heat Flow from Surfaces. PhD Thesis, University of Warwick (1996).

[21] C.-Y. WANG, Bubble phenomena of certain Palais-Smale sequences from surfaces to general targets. Houston J. Math. 22 (1996) 559-590.

[22] B. WHITE, Nonunique tangent maps at isolated singularities of harmonic maps. Bull. Amer. Math. Soc. 26 (1992) 125-129.

topping@maths.warwick.ac.uk

http://www.maths. warwick.ac.uk/ topping

Mathematics Institute, University of Warwick, Coventry, CV4 7AL, U.K. 